\title{
Multilayer silicene: clear evidence of Ag-terminated bulk silicon
}

\author{
A. Curcella, ${ }^{1}$ R. Bernard, ${ }^{1}$ Y. Borensztein, ${ }^{1}$ M. Lazzeri, ${ }^{2}$ A. Resta, ${ }^{3}$ Y. Garreau, ${ }^{3,4}$ G. Prévot, ${ }^{1 *}$ \\ ${ }^{1}$ Sorbonne Universités, UPMC Univ Paris 06, CNRS-UMR 7588, Institut des NanoSciences \\ de Paris, F-75005, Paris, France
}
${ }^{2}$ Sorbonne Universités, UPMC Univ Paris 06, CNRS-UMR 7590, MNHN, IRD UMR 206, Institut de Minéralogie, de Physique des Matériaux et de Cosmochimie, F-75005, Paris, France

${ }^{3}$ Synchrotron SOLEIL, L'Orme des Merisiers Saint-Aubin - BP 4891192 Gif-sur-Yvette CEDEX

${ }^{4}$ Université Paris Diderot, Sorbonne-Paris-Cité, MPQ, UMR 7162 CNRS, Bâtiment Condorcet, Case 7021, 75205 Paris Cedex 13, France

The existence of silicite, a new allotrope of silicon based on a stacking of hexagonal silicene planes is one of the most discussed topic in the field of 2D materials. Using grazing incidence X-ray diffraction (GIXD), we have followed in situ the growth of Si films on $\operatorname{Ag}(111)$ in the low temperature growth regime (510 - $520 \mathrm{~K})$. GIXD experiments demonstrate that Si films have a diamond-like structure, with an averaged lattice constant slightly different from bulk Si. The diffracted intensities associated with the Si films are well reproduced by the $\mathrm{Ag} / \mathrm{Si}(111)(\sqrt{3} \times \sqrt{3}) R 30^{\circ}$ honeycomb chain model, whereas models with Ag-free Si surfaces fail to reproduce the experimental data.

\footnotetext{
*Corresponding author: prevot@insp.jussieu.fr
} 
Recent years have shown an increasing interest in two-dimensional (2D) atomic layers, like graphene, silicene, germanene or phosphorene, metal dichalcogenides or trichalcogenides, or boron nitrides, which display very peculiar and promising properties $[1,2]$. Whereas many of these $2 \mathrm{D}$ materials can be obtained by exfoliation of the bulk material, this is not the case for silicene, the graphene counterpart for silicon, since no graphite analogue exists for silicon. However, it has been shown theoretically that a self-standing single layer of silicene is a metastable structure, [3-5] the binding energy of Si atoms in silicene being $0.76 \mathrm{eV}$ higher than in Si bulk.[5]

Epitaxial silicene layers have been first observed on $\operatorname{Ag}(111) .(4 \times 4),(\sqrt{13} \times \sqrt{13}) \mathrm{R} 13.9^{\circ}$ and $(2 \sqrt{3} \times 2 \sqrt{3}) \mathrm{R} 30^{\circ}$ structures have been found to coexist on the surface, with a relative proportion depending on the preparation conditions.[6-9] All those structures exhibit a buckled hexagonal organization,[10] with a buckling depending on the matching between the Si and Ag lattices. We recall that $(4 \times 4),(\sqrt{13} \times \sqrt{13}) \mathrm{R} 13.9^{\circ}$ and $(2 \sqrt{3} \times 2 \sqrt{3}) \mathrm{R} 30^{\circ}$ refers to reconstructions with respect to the $\operatorname{Ag}(111)$ surface. With respect to a free-standing silicene plane, the $(4 \times 4)$ phase corresponds to a $(3 \times 3)$ reconstruction, whereas the $(\sqrt{13} \times \sqrt{13}) \mathrm{R} 13.9^{\circ}$ and $(2 \sqrt{3} \times 2 \sqrt{3}) \mathrm{R} 30^{\circ}$ phases, to various $(\sqrt{7} \times \sqrt{7}) \mathrm{R} 19.1^{\circ}$ reconstructions. The structure and properties of these single layers are still investigated [11$14]$.

Above a coverage $\theta_{S i}=1$ monolayer (ML), a new reconstruction forms, with an apparent $(4 / \sqrt{3} \times 4 / \sqrt{3})$ unit cell with respect to the $\operatorname{Ag}(111)$ substrate [15-19]. In the following, $1 \mathrm{ML}$ corresponds to the saturation of the $(4 \times 4)$ layer, i.e. $18 / 16$ of the atomic density of a $\operatorname{Ag}(111)$ 
plane. This is only $0.8 \%$ less than the atomic density of a $\mathrm{Si}(111)$ double plane in bulk silicon. The $(4 / \sqrt{3} \times 4 / \sqrt{3})$ reconstruction remains visible even for 16 ML deposits.[20] Relatively to the silicene plane, it corresponds to a $(\sqrt{3} \times \sqrt{3}) R 30^{\circ}$ reconstruction.

These thick $\mathrm{Si} / \operatorname{Ag}(111)$ films have been firstly described as a new class of material, silicite, the equivalent of graphite for silicon atoms. This conclusion was supported by the fact that (i) the $(\sqrt{3} \times \sqrt{3}) R 30^{\circ}$ reconstruction does not exist on a clean $\mathrm{Si}(111)$ surface, (ii) the Raman signature of these thick Si films is different from the one of Si(111), and (iii) the Si interlayer spacing is $1 \%$ smaller than in the Si bulk.[20] Atomic models based on density functional theory (DFT) calculations have been proposed as alternatives of the classical diamond structure of Si, showing pseudo-layered materials with an interlayer spacing of $0.495 \mathrm{~nm}$ [21] or $0.429 \mathrm{~nm}$.[22] Models have been also proposed for the $(\sqrt{3} \times \sqrt{3}) R 30^{\circ}$ reconstruction of a silicene layer,[23] based on the initial attribution of this structure to a silicene single layer,[24,25] and for freestanding and $\mathrm{Ag}(111)$-supported silicene bilayers.[26-29] However, these results have been put into questions by further experimental studies performed by independent groups. Low-energy electron diffraction (LEED) experiments have been performed on Si thick films grown at $T_{\text {growth }}=500 \mathrm{~K}$. It was shown that the experimental data could not be explained by proposed models that only considered buckling of the Si atoms, instead a good agreement was obtained by considering a thin film of bulklike silicon terminated with the $\mathrm{Ag} / \mathrm{Si}(\sqrt{3} \times \sqrt{3}) R 30^{\circ}$ surface.[30,31] The same conclusion was put forward on the basis of a comparison between STM measurements performed on $\mathrm{Si} / \mathrm{Ag}(111)$ and on $\mathrm{Ag} / \mathrm{Si}(111)$ films in the $473 \mathrm{~K}-623 \mathrm{~K}$ temperature range,[32] and by our own experimental results: from Auger electron spectroscopy (AES) and optical reflectance measurements, we have shown that the optical response of thick ( $>10$ layers) $\mathrm{Si} / \mathrm{Ag}(111)$ films grown at $T_{\text {growth }}=473 \mathrm{~K}$ or $503 \mathrm{~K}$ was similar to the one of diamond-like bulk Si, and 
that $\mathrm{Ag}$ atoms were segregated at the surface during growth.[33] The $\mathrm{ABC}$ stacking of $\mathrm{Si}$ double planes, similar to the one of diamond-like Si has been also evidenced by STM,[34] whereas the Ag termination of the surface has been confirmed by core-level photoemission spectroscopy (XPS) measurements $\left(T_{\text {growth }}=503 \mathrm{~K}\right),[35]$ angle-resolved photoemission spectroscopy (ARPES) measurements (473 $\left.\mathrm{K} \leq T_{\text {growth }} \leq 543 \mathrm{~K}\right)$,[36] metastable atom electron spectroscopy $\left(T_{\text {growth }}=500 \mathrm{~K}\right),[37]$ and by deuterium exposure of the surface leading to the formation of $\mathrm{Ag} 3 \mathrm{D}$ islands $\left(450 \mathrm{~K} \leq T_{\text {growth }} \leq 550 \mathrm{~K}\right)$. [38]

The exact structure and surface termination of the thin Si films is therefore still discussed. Although, from most of the experimental results, the $(\sqrt{3} \times \sqrt{3}) R 30^{\circ}$ reconstruction is interpreted as due to the presence of $\mathrm{Ag}$ atoms at the surface, a reconstruction involving only silicon atoms has been put forward on the basis of scanning tunneling spectroscopy (STS) measurements [34] and STM measurements during peeling of the surface at 77 K.[39] Models for a Ag free $-(\sqrt{3} \times \sqrt{3}) R 30^{\circ}$ reconstruction of Si diamond-like films have been proposed on the basis of DFT calculations.[40,41] Other differences between $\mathrm{Si} / \mathrm{Ag}(111)$ films and $\mathrm{Ag} / \mathrm{Si}(111)$ have been also underlined: it was observed that, whereas for $\mathrm{Ag} / \mathrm{Si}(111)$ a phase transition is observed at $150 \mathrm{~K}$ between the honeycomb-chained triangle (HCT) structure and the inequivalent triangle (IET) structure,[42] a phase transition is observed at much lower temperature on $\mathrm{Si} / \mathrm{Ag}(111)$, namely at $40 \mathrm{~K} .[25,34]$

In a recent publication, De Padova et al. propose a new interpretation of the experimental discrepancies observed between the different groups [43]. Using ex situ energy dispersive grazing incidence x-ray diffraction (ED-GIXD), De Padova et al. have measured the lattice constant of $10 \mathrm{ML}$ thick Si films grown at either $473 \mathrm{~K}$ or $573 \mathrm{~K}$. Diamond-like $\mathrm{Si}$ is observed after growth at $T_{\text {growth }}=573 \mathrm{~K}$. Instead, after growth at $T_{\text {growth }}=473 \mathrm{~K}$, the diffraction pattern shows that the in-plane lattice constant of the Si film is $6.477 \AA$, i.e. $2.7 \%$ 
lower than the value found for the $(\sqrt{3} \times \sqrt{3}) R 30^{\circ}$ reconstruction of $\mathrm{Ag} / \mathrm{Si}(111)(6.655 \AA)$. In their measurements, the out-of-plane lattice constant displays also a weaker contraction, in the order of $1.5 \%$. They conclude that the crystal structure of the Si film differs from the one of diamond and that multilayer silicene forms for low temperature growth, whereas deposition at higher temperatures leads to the formation of $3 \mathrm{D}$ Si crystallites. The $(\sqrt{3} \times \sqrt{3}) R 30^{\circ}$ structure observed would thus be different for the two cases. Whereas it would correspond to the $\mathrm{Ag} / \mathrm{Si}(\sqrt{3} \times \sqrt{3}) R 30^{\circ}$ reconstruction for high temperature growth, it would be intrinsic to the pristine silicene multilayer for low temperature growth.

Indeed, it has been shown that the mode of growth and the structure of silicene single and multi-layers depend strongly on the temperature during deposition.[7,8,44,45] The $(4 \times 4)$ and $(\sqrt{13} \times \sqrt{13})$ R13.9 silicene phases preferentially form at low temperature, whereas the $(2 \sqrt{3} \times 2 \sqrt{3}) \mathrm{R} 30^{\circ}$ phase is generally seen for growth at $T_{\text {growth }}>540 \mathrm{~K}$. [8,7,6] This latter temperature also corresponds to a transition observed during multilayer growth. In fact, above $T_{\text {growth }}=540 \mathrm{~K}$, the growth of the second silicon layer is accompanied by a dewetting of the first layer, leading to the recovery of bare $\mathrm{Ag}$ surface regions. $[8,38,39,46]$ On the contrary, the silicene monolayer does not dewet below $T_{\text {growth }}=540 \mathrm{~K}$, but is progressively replaced by the growing film. $[17,19,20,33]$

Thus, if, following De Padova and collaborators, one assumes that there exist a transition temperature for the structure of thick Si films grown on $\operatorname{Ag}(111)$, it is reasonable to assign this temperature to the transition temperature $T_{c}=540 \mathrm{~K}$ above which the $(2 \sqrt{3} \times 2 \sqrt{3}) \mathrm{R} 30^{\circ}$ silicene phase forms and dewets upon further growth. However, open questions remain on the exact nature of the Si films, formed at low temperature, which are claimed to be multilayer silicene. De Padova et al. have determined the lattice constants of the films, but their atomic structure remains unknown.[20,43] The origin of the differences observed by Raman and X- 
ray diffraction (XRD) between $\mathrm{Si} / \mathrm{Ag}$ films and bulk Si [20] is also not fully understood, and no structural model for a Ag-free $(\sqrt{3} \times \sqrt{3}) R 30^{\circ}$ reconstruction has been experimentally validated.

Using grazing incidence X-ray diffraction (GIXD), we have followed the in situ growth of Si films on $\operatorname{Ag}(111)$ in the low temperature growth regime $(510-520 \mathrm{~K})$. The GIXD experiments demonstrate that $\mathrm{Si}$ films have a diamond-like structure, therefore unambiguously rejecting the existence of a new allotrope of $\mathrm{Si}$ for the growth of $\mathrm{Si} / \mathrm{Ag}(111)$. The diffracted intensities associated with the Si films are well reproduced by the $\mathrm{Ag} / \mathrm{Si}(111)$ HCT model, whereas models with Ag-free Si surfaces fail to reproduce the experimental data. Furthermore we measure an exhaustive set of Bragg reflections related to a diamond symmetry group $\mathrm{Fd} \overline{3} m$.

\section{Atomic structure of a thick Si film}

For $T_{\text {growth }}=520 \mathrm{~K}$, we have previously observed by GIXD that 1 ML evaporation leads to the preferential formation of the $(4 \times 4)$ silicene structure, with a small fraction of $(\sqrt{13} \times \sqrt{13})$ R13.9 domains.[10] In the present study, we have grown, at the same temperature, a thicker Si deposit, namely $8 \mathrm{ML}$, as estimated from surface differential reflectance spectroscopy measurements. We have verified, during growth, that only $(4 \times 4)$ and $(\sqrt{13} \times \sqrt{13})$ R13.9 reconstructions were visible below 1 ML coverage. After growth, a good agreement for the film thickness is found with x-ray reflectivity measurements, that can be fitted with a mean film thickness of $10.2 \mathrm{ML}$ and a roughness of $1 \mathrm{ML}$.

Fig. 1 shows various maps of the diffracted intensity after Si deposition. Crystal truncation rods (CTR) at integer values for $h$ and $k$ correspond to the diffraction from the Ag interface, and the intense spots at $(101),(202),(110),(113)$, to the Bragg diffraction conditions for the 
Ag crystal, in the surface unit cell coordinates. Other spots are visible and correspond to the diffraction from the silicon thin film. All these spots can be indexed with respect to a bulk diamond-like silicon film with (111) epitaxy. The diffraction spots from $\{111\},\{220\},\{311\}$, $\{400\},\{242\},\{333\},\{440\},\{531\}$ planes indeed verify the non-extinction conditions for the diamond structure: $(h, k, l)$ are of same parity and $h+k+l \neq 4 n+2$. This demonstrates the diamond structure of the Si films and thus, we can reject any structure different from bulk silicon. Lastly, no signal can be seen at the position of the expected spots for the $(4 \times 4)$ and $(\sqrt{13} \times \sqrt{13})$ R13.9 structures, indicating that the silicene single layer is not preserved below the film that covers the whole surface, in good agreement with STM and LEED observations for thick $\mathrm{Si} / \operatorname{Ag}(111)$ films prepared in similar conditions.[20,33] 

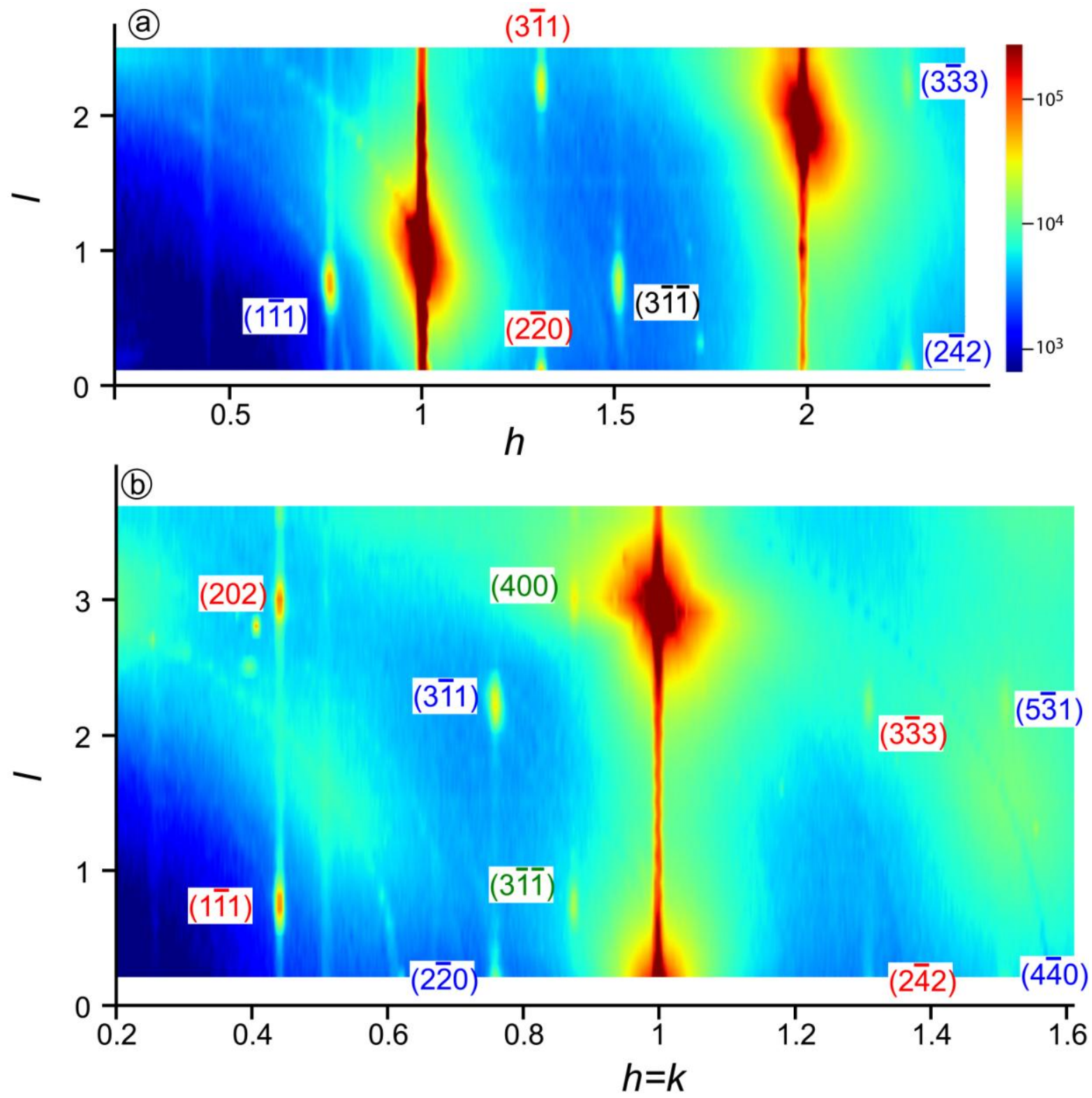

Figure 1. Maps of the intensity diffracted by $8 \mathrm{ML} \mathrm{Si}$ evaporated on $\operatorname{Ag}(111)$ at $T_{\text {growth }}=520 \mathrm{~K}$. Measurements are performed at the same temperature. a) for $k=0, \mathrm{~b}$ ) for $h=k$. The color scale is the same for both maps. The indexed spots belong to diamond-like silicon with four orientations (corresponding to blue, red, green and black labels). 
In these maps, four rotational domains are visible, corresponding to $[1 \overline{1} 0]_{\mathrm{Si}} / /[1 \overline{1} 0]_{\mathrm{Ag}}$ or $[1 \overline{1} 0]_{\mathrm{Si}} / /[1 \overline{2} 1]_{\mathrm{Ag}}$ and $\mathrm{ABC}$ or $\mathrm{ACB}$ stacking for the successive Si double planes. Rods passing through these spots can be associated with the truncation of the Si films, whereas other rods faintly visible at $(h, k) \approx(0.25,0.25)$ and $(h, k) \approx(0.5,0.5)$ for the $[1 \overline{1} 0]_{\mathrm{Si}} / /[1 \overline{1} 0]_{\mathrm{Ag}}$ epitaxy and at $(h, k) \approx(0.433,0)$ and $(h, k) \approx(0.866,0)$ for the $[1 \overline{1} 0]_{\mathrm{Si}} / /[1 \overline{2} 1]_{\mathrm{Ag}}$ epitaxy can be associated with the $(\sqrt{3} \times \sqrt{3}) R 30^{\circ}$ reconstruction of the Si surface. A wide azimuthal scan performed around the diffraction spot corresponding to (3i 1$)$ oriented Si planes is shown in Fig. 2. Various peaks are visible. We have fitted the signal with a combination of Voigt curves. Orientations of $0^{\circ}, 1.08^{\circ}, 10.91^{\circ}, 25.32^{\circ}, 26.54^{\circ}, 30^{\circ}$ and their symmetrical with respect to $30^{\circ}$ are obtained from the fits. Similar observations can be made around the other diffraction conditions and confirm that all orientations correspond to diamond-like Si. Such observation of multi-domain orientation is similar to the one found by LEED by Salomon et al.: angles of $3^{\circ}, 24.8^{\circ}$ and $30^{\circ}$ were measured after growth at 510 K.[19]

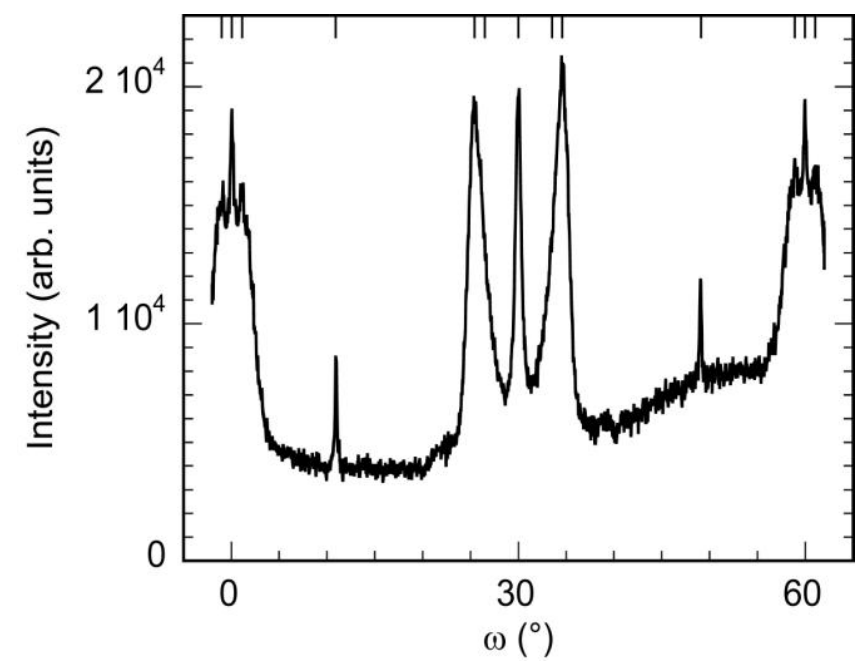

Figure 2. $\omega$-scan or azimuthal scan at a constant wave vector transfer corresponding to the diffraction by $\operatorname{Si}(3 \overline{1} 1)$ planes. The ticks on the top axis indicates the position of the fitted peaks. 
From the position of various diffraction spots, we have precisely measured the lattice constants of the Si diamond-like film which is not commensurable with the Ag surface. Whereas the ratio of the lattice constants for the Ag surface and the $(4 \times 4)$ silicene layer is exactly $3 / 4$, at this deposition temperature $(520 \mathrm{~K})$, the ratio measured for the film is $0.759 \pm 0.001$. This indicates a relaxation towards a smaller Si in-plane lattice constant when silicene transforms into bulk silicon. The Ag lattice constant at $520 \mathrm{~K}$ is $4.102 \AA$. [47] The measured Si lattice at $520 \mathrm{~K}$ is thus $5.407 \pm 0.007 \AA$. At this temperature, bulk silicon should have a lattice constant of $5.435 \AA,[48]$ and our measured constant corresponds thus to a $0.5 \%$ contraction. Once the sample has been cooled down at room temperature, an identical small contraction is observed. The lattice constant of $5.399 \pm 0.005 \AA$ measured at $300 \mathrm{~K}$ is associated with a contraction of $0.6 \%$ with respect to the lattice constant of bulk silicon $(5.431 \AA)$. We have measured the same value for all the orientations. This contraction is similar, although three times smaller, to the one observed by De Padova et al. for deposition at a lower temperature $(470 \mathrm{~K})$.

We have also obtained, at the deposition temperature, the out-of-plane lattice constant for the Si film by measuring the position along the $l$ axis of various diffraction spots. Values have bee $\mathrm{n}$ corrected for the refraction of the incoming beam through the Si film. From the position of the $(3 \overline{1} 1)$ and $(3 \overline{3} 3)$ spots at $l=l_{\operatorname{Si}(111)}=2.255$, a value of $3.140 \AA \pm 0.005 \AA$ is measured for the silicon (111) interplanar distance $d_{\mathrm{Si}(111)}$. This corresponds to the value expected for $\mathrm{Si}$ at this temperature. A nearly similar value, namely $l_{\mathrm{Si}(111)}=2.241$, corresponding to $d_{\mathrm{Si}(111)}=3.157 \AA$ is obtained from the distance along $l$ between the $(1 \overline{1} 1)$ and (202) spots and between the (3 $\overline{1} \overline{1})$ and (400) spots (see Fig. 1).

However, contrary to what is expected for a perfect FCC stacking, the (11 1$)$ and $(3 \overline{1} \overline{1})$ spots are not exactly at $l=\frac{1}{3} l_{\mathrm{Si}(111)}$ and the spots at (202) and (400) not exactly at 
$l=\frac{4}{3} l_{\mathrm{Si}(111)}$. Taking as reference the value $l_{\mathrm{Si}(111)}=2.241$ between two consecutive spots along the corresponding rods, we obtained that these spots display a positive shift $\delta l=0.035$. Such shift can be associated to the presence of stacking faults in the films. Stacking faults have been shown to shift the positions of some diffraction peaks [49]. In particular, whereas spots at $l=n l_{(111)}$ are not shifted, spots at $l=(n+1 / 3) l_{(111)}$ are shifted towards higher values, and spots at $l=(n-1 / 3) l_{(111)}$ are shifted towards lower values. This is indeed what is measured here. From the value of $\delta l$, an estimate of the stacking fault probability $p$ can be done. [50] Within the Paterson model (i.e. without correlation between faults), $\delta l / l_{\mathrm{Si}(111)}=\frac{1}{6}-\frac{1}{2 \pi} \arctan (\sqrt{3}(1-2 p))$. From this equation, we obtain that $p=0.1$, which corresponds to an average number of one stacking fault in the film, considering the mean film thickness.

Note that the measured shift could be also related to a non-negligible contribution of the surface reconstruction to the diffracted signal.

\section{Atomic structure of the $(\sqrt{3} \times \sqrt{3}) R 30^{\circ}$ reconstruction}

In a second step, we have grown, at $T_{\text {growth }}=510 \mathrm{~K}$, a $2 \mathrm{ML}$ thick $\mathrm{Si}$ film. We have followed the evolution of the diffracted signal during growth. Fig. 3 shows the evolution with coverage $\theta_{S i}$ of the diffracted intensity near the $(0.75,0.75,0.1)$ diffraction condition. Starting from a bare Ag surface, a peak corresponding to the $(4 \times 4)$ silicene superstructure is observed for coverage larger than 0.5 ML. This is in good agreement with previous STM observations showing that at low temperature, the small Si domains that form in the early stage of the growth are not ordered [51,45]. Between 0.5 ML and $1 \mathrm{ML}$, the signal increases 
abruptly, in relation with the transformation of disordered silicene regions into $(4 \times 4)$ domains.[45]

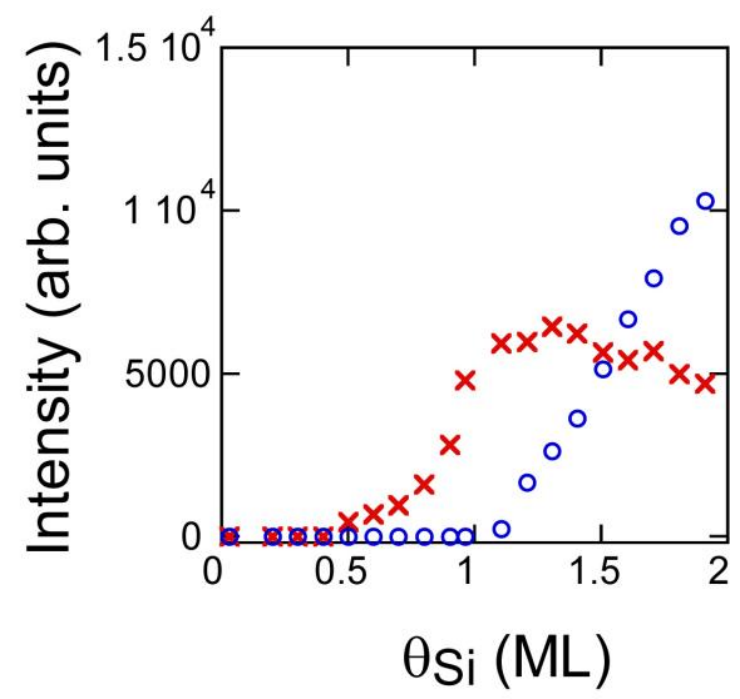

Figure 3. Evolution of the diffracted intensity for the $\left(\begin{array}{ll}0.75 & 0.750 .15)\end{array}\right)$ position (red crosses) and the $(0.760 .760 .15)$ position (blue dots), during Si evaporation at $T_{\text {growth }}=510 \mathrm{~K}$.

Above $1 \mathrm{ML}$, a second peak is visible near $(0.76,0.76,0.15)$, corresponding to the growth of the Si film. The associated intensity grows linearly with coverage, while the diffracted intensity associated with the superstructure spots $(4 \times 4)$ decay slowly. This slow decay is in good agreement with LEEM observations at 491K.[8] We recall that for the thick $8 \mathrm{ML}$ deposit, no signal could be seen for the $(0.75,0.75,0.15)$ position, indicating that the structure of the silicene single layer is not preserved below the film. The signal observed in the early stage of growth is thus related to parts of the surface which remain covered by the silicene single layer.

Fig. 4 shows the in-plane diffracted signal around the $(0.75,0.75,0.05)$ condition for $2 \mathrm{ML}$ deposition. The spots corresponding to the $(4 \times 4)$ and $(\sqrt{13} \times \sqrt{13})$ reconstructions remains visible, which confirms that in this low temperature regime, there is no dewetting of the 
silicene layer above $1 \mathrm{ML}$. The most intense spots correspond to the thin Si(111) film, with a main epitaxial relationship of $30^{\circ}$ between the Si and Ag lattice, and the less intense spots, to other domains at $\pm 4.3^{\circ}$ from the main orientation. A wider, less intense, peak is also present at $\pm 2.7^{\circ}$. More generally, we recover nearly the same orientations as the one found for the $8 \mathrm{ML}$ film, excepted the orientation previously observed at $10.91^{\circ}$. From Fig. 4 , it is clear that the $\mathrm{Si}(111)$ film has a lattice constant smaller than the one of silicene. At room temperature, we have measured, for the thin film, a Si lattice constant of $5.354 \pm 0.007 \AA$, a value $1.4 \%$ smaller than the Si bulk lattice constant $(5.431 \AA)$. This reduction is twice the value found for the 8 ML film, which indicates that the in-plane lattice constant of the film depends on the thickness and/or on the small growth temperature difference $(510 \mathrm{~K}$ vs $520 \mathrm{~K})$. From the width of the Bragg spots of the Si film in the $l$ direction, we obtain that the diffracting domains have an average thickness of 4 atomic diamond double planes. As the average deposited coverage is $2 \mathrm{ML}$, this indicates that the Si film is inhomogeneous, having regions with $1 \mathrm{ML}$ and regions with a higher number of layers. This is confirmed by the observed small decay of the intensity diffracted by the $(4 \times 4)$ domains. Only a small fraction of the silicene monolayer has been converted to thicker islands, with a height distribution that is not directly accessible with GIXD. This is in good agreement with STM observations performed on 2.5 ML thick films grown at $490 \mathrm{~K}$ where Si islands with various heights coexist with the silicene layer at the surface of the $\operatorname{Ag}(111)$ sample.[17] 


\section{0}

4000
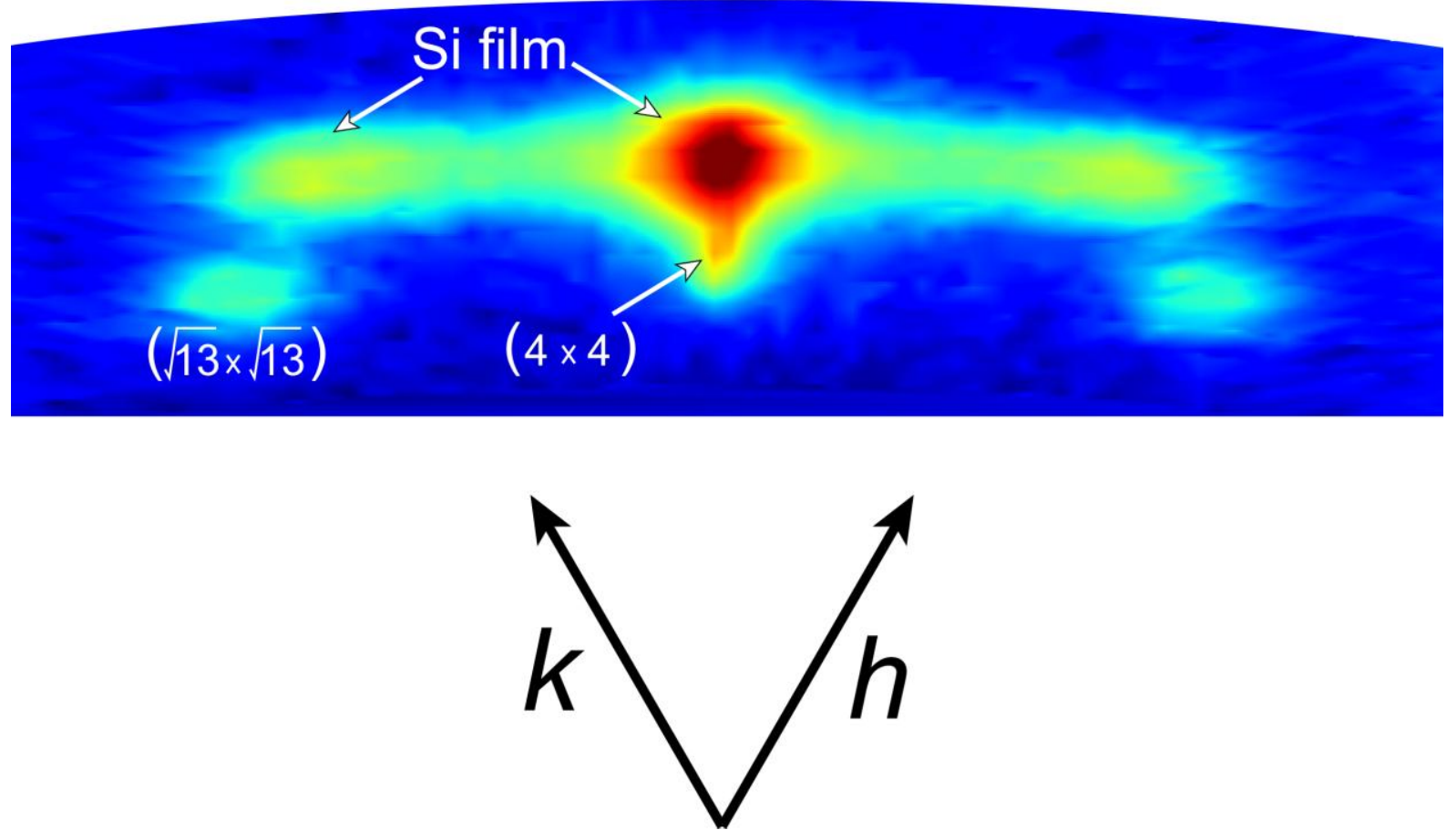

Fig. 4. in-plane map of the diffracted intensity near the $(3 / 4,3 / 4)$ spot for $l=0.05$, for $2 \mathrm{ML}$ Si deposited at $510 \mathrm{~K}$.

On this thin Si film, we have measured the structure factors associated with the $(\sqrt{3} \times \sqrt{3}) R 30^{\circ}$ reconstruction of the Si islands. For this purpose, we have performed, for inplane conditions, standard rocking scans at $l=0.05$ along several spots corresponding to the $(\sqrt{3} \times \sqrt{3}) R 30^{\circ}$ superstructure, and for "out of plane" conditions, standard rocking scans along four superstructure rods $(\mathrm{SR})$, corresponding to $(h, k) \approx(0.254,0.254), \quad(h, k) \approx(0.509,0.509)$, and $(h, k) \approx(1.27,1.27)$ and along two Si CTRs at $(h, k) \approx(0.763,0.763)$ and $(h, k) \approx(1.53$, 1.53). In the $\operatorname{Si}(\sqrt{3} \times \sqrt{3}) R 30^{\circ}$ basis, these rods are indexed as $(10 L),(20 L),(30 L),(50 L)$ 
and $(60 L)$, with $L=\frac{4}{3} l$. The standard instrumental correction was applied to the structure factors for taking into account the geometry of the diffractometer and the sample dimensions.[52]

The experimental structure factors have been further compared with simulated ones derived from the different models proposed in the literature. These models are the Ag-terminated honeycomb chain triangle model (HCT) [53], a similar model with Si trimers but without Ag atoms [31] (SiT), a model with $\mathrm{Si}$ vacancies $(\mathrm{SiV})$, proposed in the past by Fan et al. for a $(\sqrt{3} \times \sqrt{3}) R 30^{\circ}$ reconstruction observed on pristine sputter-annealed $\mathrm{Si}(111)$ [54], the model of Guo and Oshiyama of a buckled hexagonal surface termination (Hex)[55], and models of surfaces terminated with one (TDS) or two (HDS) Si adatoms per unit cell, in dumbbell positions.[23] These different models are shown in Fig. 5.

For the calculation of structure factors, we have used the ROD program with LevenbergMarquardt and simulated annealing procedures.[56] As free parameters, we have used two scale factors (for CTRs and superstructure rods respectively), Debye-Waller factors for the different layers, and in-plane and out-of plane relaxations of all the atoms in the first three silicene layers, taking into account the symmetry of the models. The agreement between experimental $\left(F_{\text {exp }}\right)$ and simulated $\left(F_{\text {th }}\right)$ structure factors is estimated from the value of $\chi^{2}=\frac{1}{N_{\mathrm{pt}}-N_{\mathrm{par}}} \sum_{\mathrm{pt}}^{N_{\mathrm{t}}}\left(\frac{F_{\mathrm{th}}-F_{\mathrm{exp}}}{\sigma_{\exp }}\right)^{2}$, where $N_{\mathrm{pt}}=171$ is the number of experimental structure factors, and $N_{\text {par }}$ the number of free parameters. The results of the fits are given in table 1. 


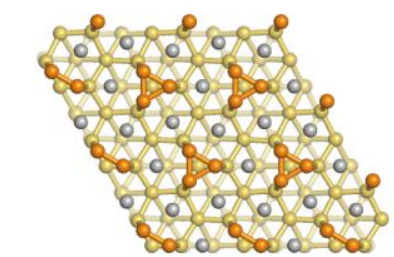

$\mathrm{HCT}$
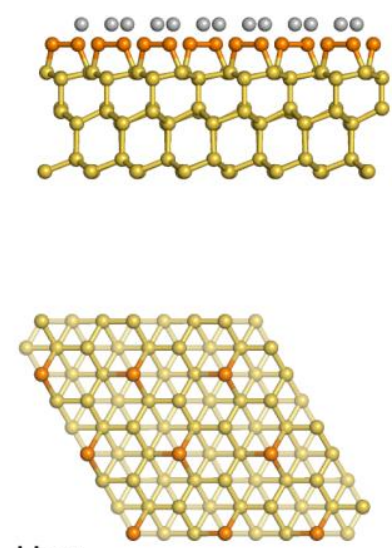

Hex

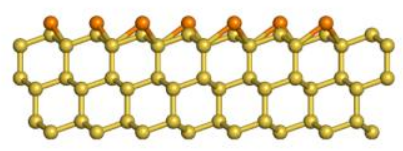

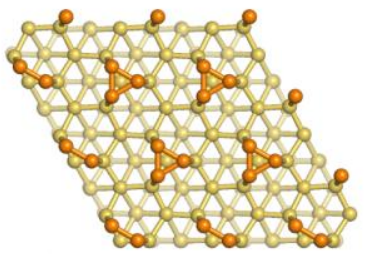

SiT
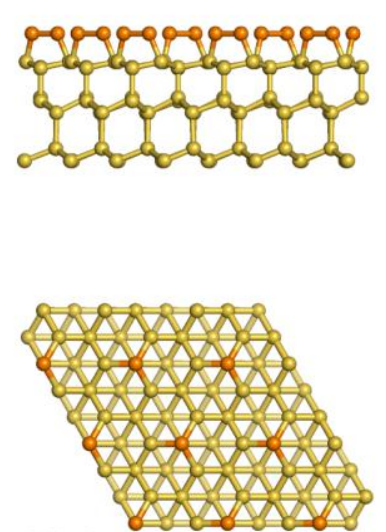

TDS

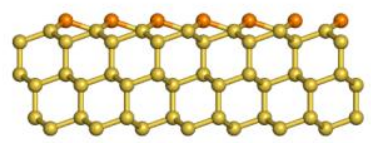

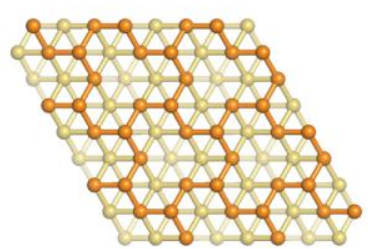

SiV
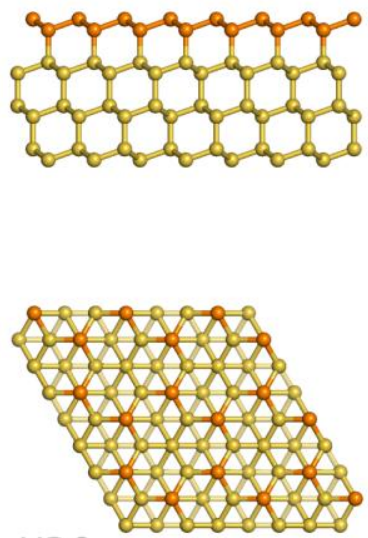

HDS

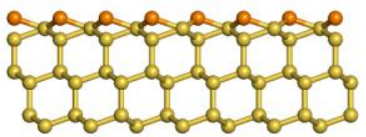

Fig. 5. Schematic views (top and lateral) of the different atomistic models used for fitting the GIXD data (honeycomb chain triangle model, Si trimers, Si vacancies, buckled hexgonal, trigonal dumbbell structure and hexagonal dumbbell structure). Ag atoms are drawn in grey. For clarity, Si atoms of the top layer are drawn in orange, while the other Si atoms are drawn in yellow. For the HCT model, the atomic positions correspond to the best fit of the data, while they correspond to the starting positions for the fit for the other models.

Whereas a very good agreement, with $\chi^{2}=3.7$, is obtained with the HCT model, i.e. with a film surface similar to the $(\sqrt{3} \times \sqrt{3}) R 30^{\circ}$ reconstruction of $\mathrm{Ag} / \mathrm{Si}(111)$, a very poor agreement is obtained with all other models, with $13.8<\chi^{2}<25.4$. The comparison between experimental and simulated structure factors along the different rods is shown in Fig. 6 for the 
best (HCT) model found and for the least worst (HDS) of the Ag-free models. While the intensity variations of the superstructure rods are well reproduced with the HCT model, the fit based on the HDS model gives a poor agreement and displays strong oscillations that are not observed experimentally. For the $(30 L)$ CTR rod, the peak at $L=3$ reflects the FCC stacking of diamond-like silicon and is thus reproduced by the two models. The spurious oscillation visible at $L=1.5$ on the fit of the latter mentioned for the HCT model is due to the interference pattern between the surface and the interface of the film. It would be suppressed by taking into account the distribution of film thicknesses. Finally, none of the Ag-free models are able to reproduce the high intensity of the $(20 L) \operatorname{rod}$ at $L=0$. This is easily explained by the fact that the atomic scattering factor $f$ depends strongly on the atomic number. For example, for (200) diffraction condition, $f_{A g}=39.8$ whereas $f_{S i}=10.2$. Thus, it is unrealistic to fit the experimental structure factors along the reconstruction rods with models that do not take into account the presence of Ag atoms.

\begin{tabular}{|l|l|}
\hline Model & $\chi^{2}$ \\
\hline HCT [53] & 3.7 \\
\hline SiT [57] & 16.3 \\
\hline SiV [54] & 25.4 \\
\hline Hex [55] & 20.4 \\
\hline TDS [23] & 18.0 \\
\hline HDS [23] & 13.8 \\
\hline
\end{tabular}

Table 1: optimized $\chi^{2}$ for the various models of $(\sqrt{3} \times \sqrt{3}) R 30^{\circ}$ reconstruction. 
Finally, we have simulated the diffracted intensities along several CTRs for a $8 \mathrm{ML}$ film terminated with the HCT reconstruction. The comparison with the experimental results show that the $(\sqrt{3} \times \sqrt{3}) R 30^{\circ}$ reconstruction of the surface cannot by itself reproduce the observed shifts of the Si Bragg peaks, that must be thus attributed to stacking faults.
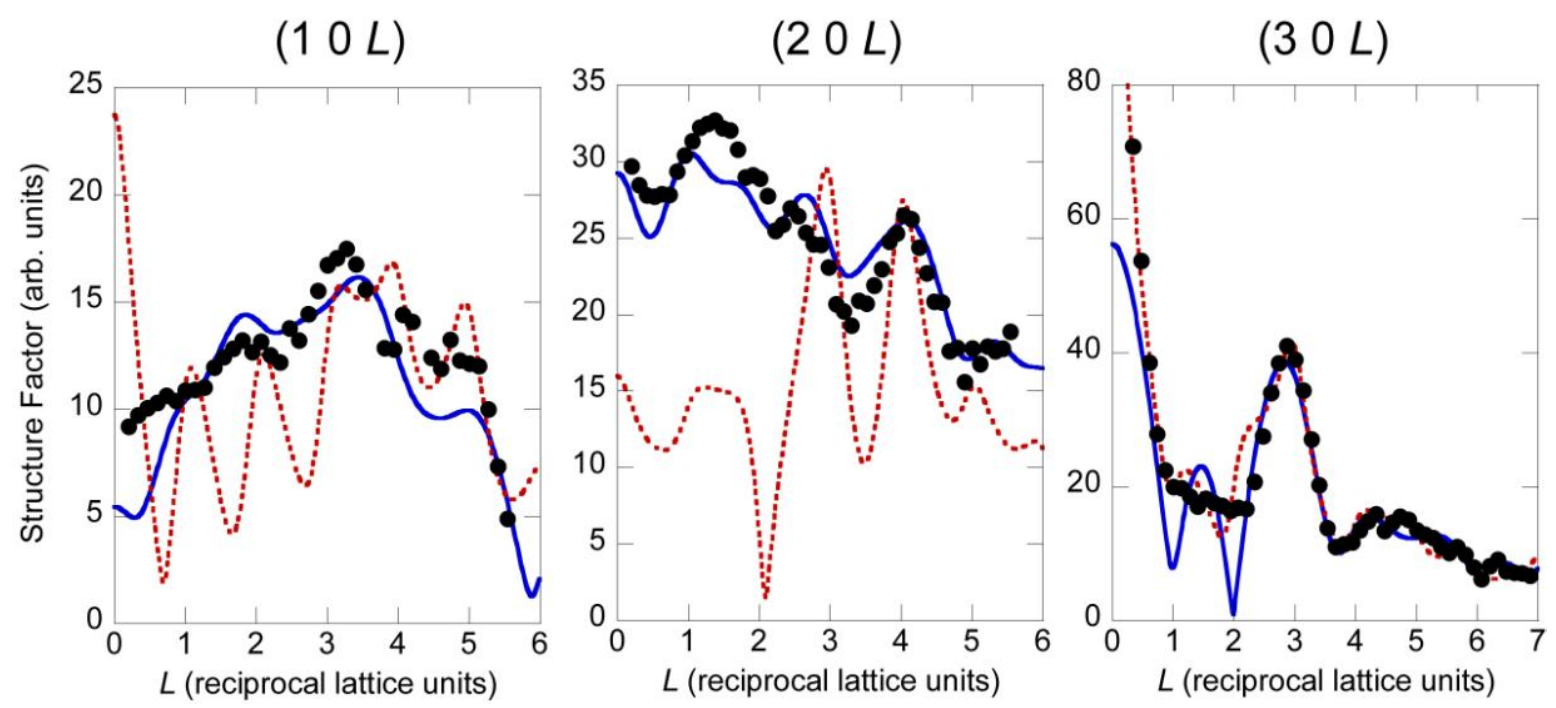

Fig. 6. Comparison between experimental (black dots) and simulated structure factors for three rods. Blue continuous line: HCT model, Red dotted line: HDS model.

\section{Discussion}

GIXD is a unique technique that allows to simultaneously measure, in situ, the structure of the bulk and the surface of a growing film. Whereas De Padova et al. have hypothesized the existence of a low temperature and a high temperature growth regime to explain the inconsistency between their results and the results of other groups, our observations demonstrate that, also for low temperature deposition conditions, i.e. for $T_{\text {growth }}<540 \mathrm{~K}$, the so-called "multilayer silicene" is in fact a thin diamond-like Si film terminated by the $\mathrm{Ag} / \mathrm{Si}(111)(\sqrt{3} \times \sqrt{3}) R 30^{\circ}$ reconstruction. Thanks to the precision of present measurements, we can provide explanations for the difference observed between high and low temperature $\mathrm{Si}$ 
growth. The Si crystal quality obviously depends on the growth temperature. Perfect Si nanocrystals are obtained for $573 \mathrm{~K}$ deposition,[43] with a lattice constant equal to the one of bulk Si. Note that this temperature corresponds also to the condition of a perfect Ag surfactant growth for the homoepitaxy of $\mathrm{Si} / \mathrm{Si}(111)$.[58] At 510-520 K, we also observe the formation of diamond-like Si, but with a lattice constant smaller than the one of bulk Si. At $470 \mathrm{~K}$, the lattice constant, measured by De Padova et al. is even smaller. Instead of being related to the formation of a new crystal structure, these lattice constant variations could be related to an increasing number of defects in the film structure as the growth temperature is lowered. At $510-520 \mathrm{~K}$, from the shift of the Bragg reflections at $l=(n+1 / 3) l_{(111)}$ and $l=(n-1 / 3) l_{(11)}$, we have concluded to the presence of one stacking fault in average in the film. It has been also theoretically shown that the in-plane lattice constant of a Si film depends on the stacking sequence of the successive planes.[59] In particular, $10 \mathrm{ML}$ thick Si films with wurtzite structure $(\mathrm{ABAB})$ have a lattice constant $6 \%$ smaller than similar films with diamond structure (ABC). [59] Thus, the presence of stacking fault could explain the smaller in-plane lattice constant measured. Finally, these results allow us to propose another interpretation for the Raman signal measured by De Padova et al.[20,43]. Lattice contractions are expected to shift phonons to higher frequencies [60] therefore the $+0.6 \%$ shift observed in the $\mathrm{G}$ band, with respect to the $520 \mathrm{~cm}^{-1}$ bulk silicon can be assigned to the contracted silicon lattice present in the sample. The broad bands between 430 and $500 \mathrm{~cm}^{-1}$ observed by De Padova et al. can instead be assigned to amorphous silicon which is known to have a very similar signature.[61]

Thus, upon those new experimental data and interpretation, there is no experimental evidence for the novel atomic organization of Si named silicite in thin $\mathrm{Si} / \mathrm{Ag}$ epitaxial films. On the contrary, our results strongly suggest that an unique growth mechanism, i.e. the 
surfactant role of $\mathrm{Ag}$ atoms, is responsible for the formation of Si diamond-like films in the $470 \mathrm{~K}-570 \mathrm{~K}$ temperature range on $\operatorname{Ag}(111)$.

\section{METHODS}

GIXD experiments have been performed on the SIXS beamline of SOLEIL synchrotron. The $\operatorname{Ag}(111)$ sample was prepared by repeated cycles of $\mathrm{Ar}^{+}$sputtering and annealing at $800 \mathrm{~K}$. Si was evaporated in the diffraction chamber (base pressure $4.10^{-10}$ Torr) from a thermally heated crucible using a commercial Omicron Nanotechnology e-beam evaporator. From surface differential reflectance spectroscopy measurements, we estimated that the deposition rate was $1 \mathrm{ML} / 2700 \mathrm{~s}$. The sample was analyzed with $18.46 \mathrm{keV}$ X-rays at a grazing incidence angle of $0.145^{\circ}$. Diffracted X-rays were detected with a point detector. The $(h, k, l)$ indices used for indexing a reflection in reciprocal space refer to the $\operatorname{Ag}(111)$ surface basis $\left(a=b=2.889 \AA, c=7.075 \AA, \alpha=\beta=90^{\circ}, \gamma=120^{\circ}\right)$. The $(H, K, L)$ indices refer to the $\operatorname{Si}(\sqrt{3} \times \sqrt{3}) R 30^{\circ}$ reconstruction with $\left(a=b=a_{0 S i} \sqrt{3 / 2}, c=a_{0 S i} \sqrt{3}, \alpha=\beta=90^{\circ}, \gamma=120^{\circ}\right)$.

\section{ACKNOWLEDGMENTS}

This work was supported by French state funds managed by the ANR within the Investissements d'Avenir program under Reference ANR-11-IDEX-0004-02 and more specifically within the framework of the Cluster of Excellence MATISSE. The SIXS beamline staff is greatly acknowledged for the technical support.

\section{REFERENCES}

[1] Geim A K and Grigorieva I V 2013 Van der Waals heterostructures Nature 499 419-25

[2] Butler S Z, Hollen S M, Cao L, Cui Y, Gupta J A, Gutiérrez H R, Heinz T F, Hong S S, Huang J, Ismach A F, Johnston-Halperin E, Kuno M, Plashnitsa V V, Robinson R D, 
Ruoff R S, Salahuddin S, Shan J, Shi L, Spencer M G, Terrones M, Windl W and Goldberger J E 2013 Progress, Challenges, and Opportunities in Two-Dimensional Materials Beyond Graphene ACS Nano 7 2898-926

[3] Takeda K and Shiraishi K 1994 Theoretical possibility of stage corrugation in Si and Ge analogs of graphite Phys. Rev. B 5014916

[4] Guzmán-Verri G and Lew Yan Voon L 2007 Electronic structure of silicon-based nanostructures Phys. Rev. B 76075131

[5] Cahangirov S, Topsakal M, Aktürk E, Şahin H and Ciraci S 2009 Two- and OneDimensional Honeycomb Structures of Silicon and Germanium Phys. Rev. Lett. 102 236804

[6] Vogt P, De Padova P, Quaresima C, Avila J, Frantzeskakis E, Asensio M C, Resta A, Ealet B and Le Lay G 2012 Silicene: Compelling Experimental Evidence for Graphenelike Two-Dimensional Silicon Phys. Rev. Lett. 108155501

[7] Jamgotchian H, Colignon Y, Hamzaoui N, Ealet B, Hoarau J Y, Aufray B and Bibérian J P 2012 Growth of silicene layers on $\operatorname{Ag}(111)$ : unexpected effect of the substrate temperature J. Phys. Condens. Matter 24172001

[8] Moras P, Mentes T O, Sheverdyaeva P M, Locatelli A and C Carbone 2014 Coexistence of multiple silicene phases in silicon grown on $\operatorname{Ag}\left(\begin{array}{lll}1 & 1 & 1\end{array}\right)$ J. Phys. Condens. Matter 26 185001

[9] Grazianetti C, Chiappe D, Cinquanta E, Fanciulli M and Molle A 2015 Nucleation and temperature-driven phase transitions of silicene superstructures on $\operatorname{Ag}\left(\begin{array}{lll}1 & 1 & 1\end{array}\right)$ J. Phys. Condens. Matter 27255005

[10] Curcella A, Bernard R, Borensztein Y, Resta A, Lazzeri M and Prévot G 2016 Determining the atomic structure of the $(4 \times 4)$ silicene layer on $\operatorname{Ag}(111)$ by combined grazing-incidence $\mathrm{x}$-ray diffraction measurements and first-principles calculations $P$ hys. Rev. B 94165438

[11] Lew Yan Voon L C, Zhu J and Schwingenschlögl U 2016 Silicene: Recent theoretical advances Appl. Phys. Rev. 3040802

[12] Zhao J, Liu H, Yu Z, Quhe R, Zhou S, Wang Y, Liu C C, Zhong H, Han N, Lu J, Yao Y and Wu K 2016 Rise of silicene: A competitive 2D material Prog. Mater. Sci. 83 24-151

[13] Grazianetti C, Cinquanta E and Molle A 2016 Two-dimensional silicon: the advent of silicene 2D Mater. 3012001

[14] Takagi N, Lin C-L, Kawahara K, Minamitani E, Tsukahara N, Kawai M and Arafune R 2015 Silicene on $\operatorname{Ag}(111)$ : Geometric and electronic structures of a new honeycomb material of Si Prog. Surf. Sci. 90 1-20

[15] De Padova P, Vogt P, Resta A, Avila J, Razado-Colambo I, Quaresima C, Ottaviani C, Olivieri B, Bruhn T, Hirahara T, Shirai T, Hasegawa S, Carmen Asensio M and Le Lay G 2013 Evidence of Dirac fermions in multilayer silicene Appl. Phys. Lett. 102163106 
[16] De Padova P, Avila J, Resta A, Razado-Colambo I, Quaresima C, Ottaviani C, Olivieri B, Bruhn T, Vogt P, Asensio M C and Le Lay G 2013 The quasiparticle band dispersion in epitaxial multilayer silicene J. Phys.-Condens. Matter 25382202

[17] Vogt P, Capiod P, Berthe M, Resta A, De Padova P, Bruhn T, Le Lay G and Grandidier B 2014 Synthesis and electrical conductivity of multilayer silicene Appl. Phys. Lett. 104 021602

[18] Sone J, Yamagami T, Aoki Y, Nakatsuji K and Hirayama H 2014 Epitaxial growth of silicene on ultra-thin Ag(111) films New J. Phys. 16095004

[19] Salomon E, Ajjouri R E, Lay G L and Angot T 2014 Growth and structural properties of silicene at multilayer coverage J. Phys. Condens. Matter 26185003

[20] De Padova P, Ottaviani C, Quaresima C, Olivieri B, Imperatori P, Salomon E, Angot T, Quagliano L, Romano C, Vona A, Muniz-Miranda M, Generosi A, Paci B and Le Lay G 201424 h stability of thick multilayer silicene in air 2D Mater. 1021003

[21] Wang Y, Scheerschmidt K and Gösele U 2000 Theoretical investigations of bond properties in graphite and graphitic silicon Phys. Rev. B 6112864

[22] Cahangirov S, Özçelik V O, Rubio A and Ciraci S 2014 Silicite: The layered allotrope of silicon Phys. Rev. B 90085426

[23] Cahangirov S, Özçelik V O, Xian L, Avila J, Cho S, Asensio M C, Ciraci S and Rubio A 2014 Atomic structure of the $3 \times 3$ phase of silicene on $\operatorname{Ag}(111)$ Phys. Rev. B 90 035448

[24] Chen L, Liu C-C, Feng B, He X, Cheng P, Ding Z, Meng S, Yao Y and Wu K 2012 Evidence for Dirac Fermions in a Honeycomb Lattice Based on Silicon Phys. Rev. Lett. 109056804

[25] Chen L, Li H, Feng B, Ding Z, Qiu J, Cheng P, Wu K and Meng S 2013 Spontaneous Symmetry Breaking and Dynamic Phase Transition in Monolayer Silicene Phys. Rev. Lett. 110085504

[26] Sakai Y and Oshiyama A 2015 Structural stability and energy-gap modulation through atomic protrusion in freestanding bilayer silicene Phys. Rev. B 91201405

[27] Pflugradt P, Matthes L and Bechstedt F 2014 Unexpected symmetry and AA stacking of bilayer silicene on \$ \mathrmAg(111)\$ Phys. Rev. B 89205428

[28] Ezawa M 2012 Quasi-Topological Insulator and Trigonal Warping in Gated Bilayer Silicene J. Phys. Soc. Jpn. 81104713

[29] Johnson N W, Muir D, Kurmaev E Z and Moewes A 2015 Stability and Electronic Characteristics of Epitaxial Silicene Multilayers on $\mathrm{Ag}(111)$ Adv. Funct. Mater. 25 4083-90

[30] Shirai T, Shirasawa T, Hirahara T, Fukui N, Takahashi T and Hasegawa S 2014 Structure determination of multilayer silicene grown on $\mathrm{Ag}(111)$ films by electron diffraction: Evidence for Ag segregation at the surface Phys. Rev. B 89241403 
[31] Kawahara K, Shirasawa T, Lin C-L, Nagao R, Tsukahara N, Takahashi T, Arafune R, Kawai M and Takagi N 2016 Atomic structure of "multilayer silicene" grown on $\operatorname{Ag}(111)$ : Dynamical low energy electron diffraction analysis Surf. Sci. 651 70-5

[32] Mannix A J, Kiraly B, Fisher B L, Hersam M C and Guisinger N P 2014 Silicon Growth at the Two-Dimensional Limit on Ag(111) ACS Nano 8 7538-47

[33] Borensztein Y, Curcella A, Royer S and Prévot G 2015 Silicene multilayers on $\operatorname{Ag}(111)$ display a cubic diamondlike structure and a $\sqrt{ } 3 \times \sqrt{ } 3$ reconstruction induced by surfactant Ag atoms Phys. Rev. B 92155407

[34] Fu H, Chen L, Chen J, Qiu J, Ding Z, Zhang J, Wu K, Li H and Meng S 2015 Multilayered silicene: the bottom-up approach for a weakly relaxed $\mathrm{Si}(111)$ with Dirac surface states Nanoscale 7 15880-5

[35] Lee G-W, Chen H-D and Lin D-S 2015 Bonding and interface formation for Si on $\mathrm{Ag}(111)$ by core-level photoemission spectroscopy Appl. Surf. Sci. 354 212-5

[36] Mahatha S K, Moras P, Sheverdyaeva P M, Flammini R, Horn K and Carbone C 2015 Evidence for a diamondlike electronic band structure of Si multilayers on $\mathrm{Ag}(111)$ Phys. Rev. B 92245127

[37] Lin C-L, Hagino T, Ito Y, Kawahara K, Nagao R, Aoki M, Masuda S, Arafune R, Kawai M and Takagi N 2016 Spectroscopic Identification of Ag-Terminated "Multilayer Silicene" Grown on Ag(111) J. Phys. Chem. C $1206689-93$

[38] Chen H-D, Chien K-H, Lin C-Y, Chiang T-C and Lin D-S 2016 Few-Layer Silicon Films on the $\operatorname{Ag}(111)$ Surface J. Phys. Chem. C 120 2698-702

[39] Chen J, Du Y, Li Z, Li W, Feng B, Qiu J, Cheng P, Xue Dou S, Chen L and Wu K 2015 Delocalized Surface State in Epitaxial Si(111) Film with Spontaneous $\sqrt{3} \times \sqrt{3}$ Superstructure Sci. Rep. 513590

[40] Guo Z-X and Oshiyama A 2014 Structural tristability and deep Dirac states in bilayer silicene on $\operatorname{Ag}(111)$ surfaces Phys. Rev. B 89155418

[41] Guo Z-X and Oshiyama A 2015 Crossover between silicene and ultra-thin Si atomic layers on Ag (111) surfaces New J. Phys. 17045028

[42] Tajiri H, Sumitani K, Nakatani S, Nojima A, Takahashi T, Akimoto K, Sugiyama H, Zhang X and Kawata H 2003 X-ray diffraction study of the Si ( 111 ) $-3 \times 3-$ Ag surface structure Phys. Rev. B 68035330

[43] De Padova P, Generosi A, Paci B, Ottaviani C, Quaresima C, Olivieri B, Salomon E, Angot T and Le Lay G 2016 Multilayer silicene: clear evidence 2D Mater. 3031011

[44] Lee G-W, Chen H-D and Lin D-S 2015 Growth mode and structures of silicene on the $\operatorname{Ag}\left(\begin{array}{lll}1 & 1 & 1\end{array}\right)$ surface 7th Vac. Surf. Sci. Conf. Asia Aust. VASSCAA-7 354, Part A 187-95

[45] Bernard R, Borensztein Y, Cruguel H, Lazzeri M and Prévot G 2015 Growth mechanism of silicene on $\mathrm{Ag}$ ( 111 ) determined by scanning tunneling microscopy measurements and ab initio calculations Phys. Rev. B 92045415 
[46] Acun A, Poelsema B, Zandvliet H J W and van Gastel R 2013 The instability of silicene on Ag(111) Appl. Phys. Lett. 103263119

[47] Touloukian Y S, Kirby R K, Taylor R E and Desai P D 1975 Thermophysical Properties of Matter vol 12 (New York)

[48] Yim W M and Paff R J 1974 Thermal expansion of AlN, sapphire, and silicon J. Appl. Phys. 451456

[49] Paterson M S 1952 X-Ray Diffraction by Face-Centered Cubic Crystals with Deformation Faults J. Appl. Phys. 23805

[50] Girard Y, Baudot G, Repain V, Rohart S, Rousset S, Coati A and Garreau Y 2005 Structural modifications of Co ultrathin films grown on $\mathrm{Au}(111)$ vicinal surfaces Phys. Rev. B 72155434

[51] Prévot G, Bernard R, Cruguel H and Borensztein Y 2014 Monitoring Si growth on $\operatorname{Ag}(111)$ with scanning tunneling microscopy reveals that silicene structure involves silver atoms Appl. Phys. Lett. 105213106

[52] Robach O, Garreau Y, Aïd K and Véron-Jolliot M B 2000 Corrections for surface X-ray diffraction measurements using the Z-axis geometry: finite size effects in direct and reciprocal space J. Appl. Crystallogr. 33 1006-1018

[53] Takahashi T and Nakatani S 1993 Refinement of the Si (111) 3\$ltimes\$ 3-Ag structure by surface X-ray diffraction Surf. Sci. 282 17-32

[54] Fan W C, Ignatiev A, Huang H and Tong S Y 1989 Observation and structural determination of $(\sqrt{ } 3 \$$ Itimes $\$ \sqrt{ } 3) \mathrm{R} 30^{\circ}$ reconstruction of the Si (111) surface Phys. Rev. Lett. 621516

[55] Guo Z-X and Oshiyama A 2015 Crossover between silicene and ultra-thin Si atomic layers on $\mathrm{Ag}(111)$ surfaces New J. Phys. 17045028

[56] Vlieg E 2000 ROD: a program for surface X-ray crystallography J. Appl. Crystallogr. 33 401-405

[57] Kawahara K, Shirasawa T, Arafune R, Lin C-L, Takahashi T, Kawai M and Takagi N 2014 Determination of atomic positions in silicene on $\mathrm{Ag}(111)$ by low-energy electron diffraction Surf. Sci. 623 25-8

[58] Yamagami T, Sone J, Nakatsuji K and Hirayama H 2014 Surfactant role of Ag atoms in the growth of $\mathrm{Si}$ layers on $\mathrm{Si}(111) \sqrt{3} \times \sqrt{3}-\mathrm{Ag}$ substrates Appl. Phys. Lett. 105151603

[59] Kamal C, Chakrabarti A, Banerjee A and Deb S K 2013 Silicene beyond mono-layersdifferent stacking configurations and their properties J. Phys. Condens. Matter 25 085508

[60] DeWolf I 1996 Micro-Raman spectroscopy to study local mechanical stress in silicon integrated circuits Semicond. Sci. Technol. 11 139-54 
[61] Voutsas A T, Hatalis M K, Boyce J and Chiang A 1995 Raman spectroscopy of amorphous and microcrystalline silicon films deposited by low-pressure chemical vapor deposition J. Appl. Phys. 786999 